\title{
Recognition of Amazigh Characters using Visual Rotation Invariant Features
}

\author{
Younès Raoui \\ LIMIARF, Université Mohamed V-Agdal, Faculté \\ des Sciences, Rabat
}

\author{
El Houssine Bouyakhf \\ LIMIARF, Université Mohamed V-Agdal, Faculté \\ des Sciences, Rabat
}

\begin{abstract}
In this paper, we present a new method for Amazigh Character Recognition. It is based on the extraction of salient points from the Tifinagh characters using a visual detector and descriptor. The novelty of this work is, first, the development of a new detector descriptor having a high repeatability which outperforms other transforms like (SIFT, SURF, Harris), second, its application for the recognition of the Amazigh characters. Our method is called Rotation Invariant Detector (RID). We use steerable filters for representing the image in many scales and orientations, then, the Harris detector applied to each of the obtained images, because of its high speed in the detection operation. In order to recognize a character, we match our image test to the database of learnt characters. We present in this work two applications based on RID:
\end{abstract}

- The visual recognition of handwritten Tifinagh Characters using the paradigm (learningclassification)

The conversion of a handwritten character to a typed character with a computer

\section{INTRODUCTION}

The computer vision is the science of recognizing objects in the images and interpreting them. During the last decade, this discipline has been widely investigated because of its relation to other fields such as charactere treatment and image retrieval. In order to interpret an image, the analysis has to move from image processing tasks to object recognition and categorization. Consequently, the algorithms which are used should be faster and more complicated. The description of the images can be obtained with predifined kernels to obtain edges. Or by using more recent differential methods to extract coins or feature points.

The feature points are better for object recognition. This is because, they are more distinctive and repeatable. Harris is one of the first detectors and still gaining success because of its speed (C. Harris and M. Stephens 1998). At the same time we find also SIFT (Scale Invariant Feature Transform) and SURF (Speeded Up Robust Feature), L.G.H.Bay, T.Tuytelaars (2006), detectors and descriptors actually very robust.
To recognize an object in a certain image, first we apply the algorithm which detects and describes the set of the training images. Second, we recognize the object using the matching algorithm in order to compute similarities between the image database and the test image. We are interested in this work to the optical character recognition. This concept emerged few years ago. It is a popular research area because of its utilization in several important domains such as postal automation and document analysis.

Recently, some efforts have been reported in literature for Amazigh characters recognition based on artificial neural networks (Y. Ait ouguengay, M. Taalabi, (2009)) Hidden Markov Models (Y. Es Saady, A. Rachidi, M. El Yassa, D. Mammass (1998)), and Hough transforms. We propose in this paper to use the paradigm of visual detection description with salient points to recognize Tifinagh characters and to convert them to characters typed with a computer. This is important in particular to develop modules which could be installed on popular word processing software using the visual approach.

This paper is organized as following. In the first section, we will introduce the approach of image interpretation with salient features. In the second section we present some issues about Amazigh language. In the third section we present a new approach for coins detection and description which we call Rotation Invariant Descriptor (RID). In the fourth section we present application of the developed detector descriptor on handwritten characters recognition and conversion to typed characters. Finaly we make some conclusions.

\section{THE TIFINAGH ALPHABET}

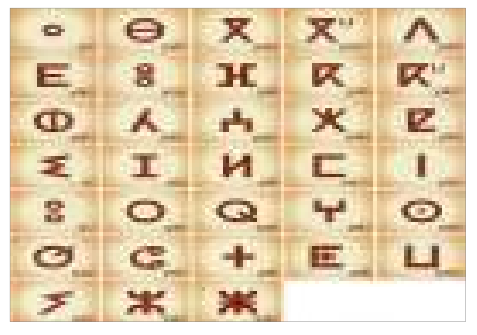

Fig.1 The Tifinagh alphabet 
The Amazigh language is spoken in many countries in Africa such as Morocco, Algeria, Tunisia and Egyptian Oasis. In Morocco, the term Berber (Amazigh) includes the three main Moroccan variants: Tarifite, Tamazighte and Tachelhite. More than $40 \%$ of the country's populations speak Berber. The establishment of The "Royal Institute of the Amazigh Culture" (IRCAM) carried out a major action to standardize the Amazigh language (Es Saady Y., Rachidi A., El Yassa M., Mammass D, 2011).The IRCAM has as main goal to regulate this language taking as central actions the language and the culture dimensions. Thus, through these procedures, the IRCAM has succeeded to save this language and to promote it. Till the beginning of the third millennium, big efforts was done in the scholar books in order to officialize this language. The Tifinagh is the writing alphabet of the Amazigh language. It has known many changes from its origin. In North Africa, an old version which dates from the 3 rd century $\mathrm{BC}$ to the 3 rd century $\mathrm{AD}$ was spoken in the Berber community.

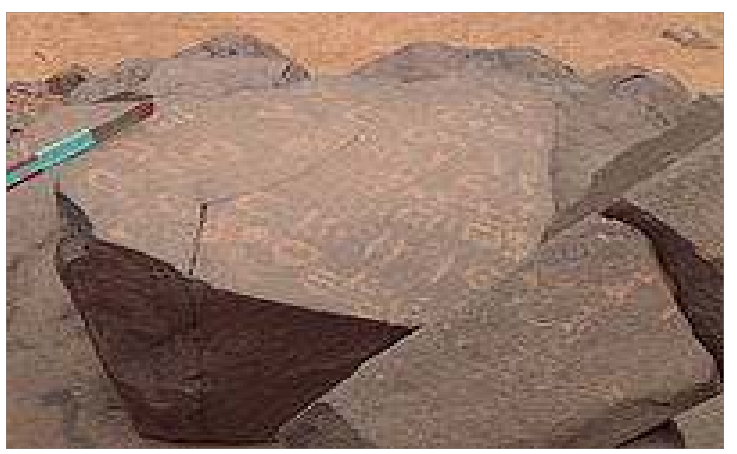

Fig2.Old tifinagh script

\section{RELATED WORK}

In the area of visual feature extraction, after the contributions of Moravec and Harris which introduced the concept of corners based on differential operators (Harris et Stephens 1998), Lowe proposed SIFT (Scale Invariant Features Transforms) to insure some of the properties like invariance to rotations, scales and illumination. This detector descriptor is based essentially on the computation of the differential of Gaussians followed with the computation of the gradient vector around each keypoint. Two years after, (L.G.H. Bay, T.Tuytelaars, 2006), developed SURF (Speeded Up Robust Feature), another detector descriptor, because SIFT was not enough repeatable, distinctive and robust to do tasks like object recognition and object categorization efficiently.

Nevertheless, these detector descriptors still have to be improved when doing tasks of recognition. Because, this requires having invariant features when the robot changes its viewpoint.

In other words, the repeatability should be better than $70 \%$ between two successive images of the same scene. We present in the next section, a new method for detector computation based on Harris detector and steerable filters. It gives a repeatability of $90 \%$.

\section{GENERATING LOCAL FEATURES}

In character processing interpretation, one of the main properties on which the computer society is focused, is the ability to keep invariance when the angle of view from which we see the character is changing (K.Mikolajzyk and C.Schmid, 2004). We propose in this paper a novel method for character detection and description which increases the repeatability rate. Our method is devided into 2 main steps :

[1] detection of feature points with an improved variant of the Harris detector

[2] description of the patch around each feature using an estimation of the color frequencies.

\subsection{Steerable filters}

Steerable filters are orientation selective convolution kernels used for feature extraction (D. Forsyth and J. Ponce 2003). The concept of steerable filters presents an efficient scheme to synthesize filters of arbitrary orientation from a linear combination of basis filters which allow steering a filter to any orientation. Consequently the filter output is determined analytically as a function of orientation.

Considering the $\mathrm{n}^{\text {th }}$ order Gaussian derivative $\mathrm{G}$ relative to an arbitrary orientation $\$ \backslash$ theta $\$$ is given the steerable filter response for the $n^{\text {th }}$ order Gaussian derivative $G_{n}(\theta)$ to an arbitrary

Orientation $\Theta$ is, the steerable filter is given with the following Gaussian distribution G:

$$
\begin{gathered}
G_{3}(\theta)=K_{31}(\theta) * G_{3}(0)+K_{32}(\theta) * G_{3}(45)+K_{33}(\theta) \\
* \mathbf{G}_{3}(90)+K_{34}(\boldsymbol{\theta}) * G_{3}(135)
\end{gathered}
$$

$$
K_{3 i}(\theta)=\frac{1}{4} * 2 * \cos \left(2\left(\theta-\theta_{i}\right)\right)+2 * \cos \left(3\left(\theta-\theta_{i}\right)\right)
$$

\section{- Detector computation:}

We apply the steerable filters described in 3.1 because they permit to represent the image at many levels of scales and rotate the image according to many orientations. Following this step, we apply the Harris detector to have $\mathrm{n}$ pyramids (with reference to $n$ orientations). Each of these pyramids has $\mathrm{s}$ levels (with reference to s scales). To cluster these features according to their positions, we use the kmean method because of its speed and its simplicity. The corresponding feature point is computed with the calculation of the mean of all features in a cluster. Thus, whatever the orientation (or the scale) of the Tifinagh characters are, the coins are the same with a very small error as shown in the fig. 4

\section{- Descriptor computation}

Around each interest point, we construct a patch then we sample the pixels in the frequency domain. In other words, we represent the patch in many quantification levels. Our idea to measure the disorder in the patch is to compute the entropy of each of these determined levels. These entropies are invariant to the changing of features such as scale and orientations. Although scale and orientation are not parts of the detector described above. 


\subsection{REPEATABILITY}

This score is used to compute the degree of invariance of the interest points when the viewpoint of the image changes (i.e. see figure $\backslash$ ref $\{$ graph $\})$. It consists to detect the interest points found in both images relative to the lowest total number of interest points. However, only the part of the image that is visible in both images is taken into account.

This ratio is defines with the following quotient:

\section{Number of similar features between the two images}

Number of the detected features in the two images

As shown in the first figure, the repeatability score varies from an angle of $921 \%$ to 74 for an angle of 50 degree.

In the second figure, the repeatability score varies from $\$ 981 \% \$$ for an angle of 20 degree to $55 \backslash \%$ for an angle of 50 .

\section{APPLICATION OF RID TO TIFINAGH CHARACTER RECOGNITION}

As explained before, the two steps for the recognition of a Tifinagh character are : Learning and Matching.

In order to learn a character, we use the database (Es Saady Y., Rachidi A., El Yassa M., Mammass D, 2011), which contains each of the Tifinagh characters written in several forms. We construct a table of the characteristic of the learnt character for $\mathrm{N}$ orientation. Each of the orientations contains all the feature points. Thus our set contains few hundreds of entries (Y. Es Saady 2012). Upon this features database, we develop two applications:

1. Character recognition: Which consists to match between this database and the table computed from the test character. To validate the distinctiveness and the efficiency of our method (i.e. RID), we do matching to many databases, each one contains a different character.

2. Conversion to typed characters by computer: This operation is based on the same method as the recognition of handwritten characters. Expect, it doesn't learn the whole forms of the characters. Instead of that, we describe the handwritten character, and we compare it with the visual descriptions of the all typed characters (the alphabet of Tifinagh). The (tab2). shows that we can retrieve the correct character from the alphabet. Then with a simple program of conversion, we can display this typed character on the electronic document .

Tab1. Recog nition of handw ritten characters : Matching between the image database and the image test using (RID)

Tab2. Transformation of a handwritten character to the typed character by computer: Matching between the Alphabet and the handwritten character is exploited the RID

\section{CONCLUSION}

In order to recognize accurately an Amazigh Character, we have proposed in this paper a new detector descriptor invariant to rotations. It allows to retrieve Tifinagh handwritten characters from a database and to retype them in a computer form. Our salient detector descriptor is based essentially on the Harris detector with our stress on the frequencies of colors within the treatedcharacter. We show that the repeatability of this new tool out-performs most of the existing features. This work is suitable for the analysis and the treatment of documents written with the Tifinagh language. They could be at the same time used in mobile applications such as Android operating systems to make people aware of this ancient culture.

\section{REFERENCES}

[1] Y. Es Saady., A. Rachidi A., M. El Yassa, D. Mammass 2011. AMHCD: A Database for Amazigh Handwritten Character Recognition Research International Journal of Computer Applications (0975 -8887) Volume 27- No.4.

[2] Y. Ait ouguengay, M. Taalabi, 2009, "Elaboration d'un réseau de neurones artificiels pour la reconnaissance optique de la graphie amazighe: Phase d'apprentissage", Systèmes intelligents-Théories et applications.

[3] Y. Es Saady, A. Rachidi, M. El Yassa, D. Mammass, 2011, "Reconnaissance Automatique de l'Ecriture Amazighe à base de Ligne Centrale de l'Écriture", 4ème Atelier international sur l'amazighe et les TIC, IRCAM, Maroc.

[4] Amrouch, A. Rachidi, M. Elyassa, D. Mammass,"Handwritten Amazigh Character Recognition, 2010, Based On Hidden Markov Models", ICGST-GVIP Journal, Vol.10,Issue 5, pp.11-18.

[5] C. Harris and M. Stephens,1988, "A combined corner and edge detector,".

[6] Y. Es Saady, 2012, Contribution au développement d'approches de reconnaissance automatique de 
[7] caractères imprimés et manuscrits, de textes et de documents, FSA, Agadir, Maroc.

[8] K.Mikolajzyk and C.Schmid, 2004, "Scale affine in technology," Proceedings of ICRA.
[9] D. Forsyth and J. Ponce, 2003, Computer Vision: A Modern Approach. Prentice-Hall.

[10] L. G. H. Bay, T.Tuytelaars, 2006, “SURF: Speeded up Robust Features," in ECCV,
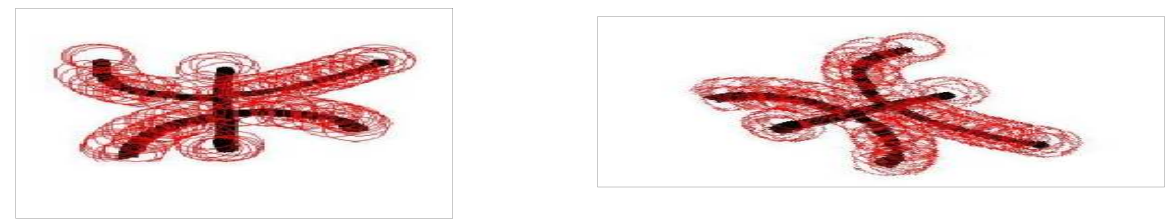

Fig3. The character written with two persons in different positions and scales

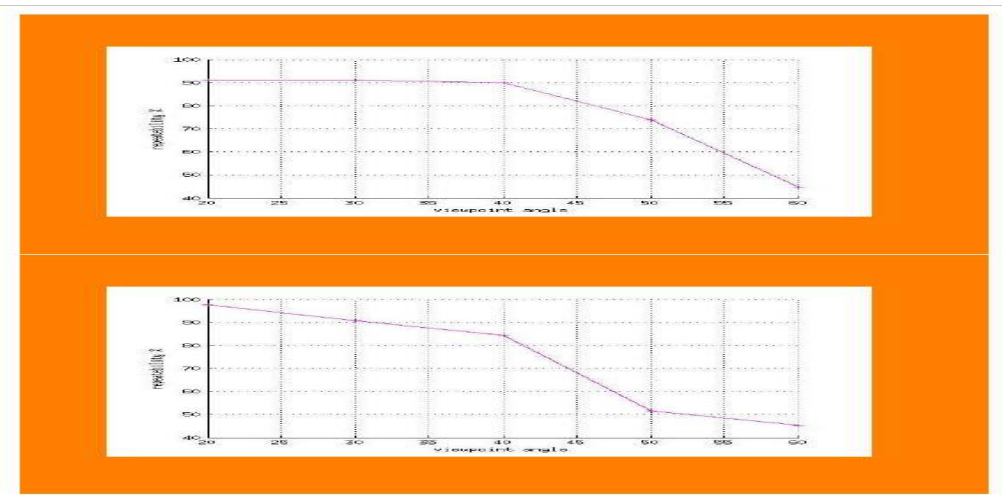

Fig4. Repeatability computed for 2 characters taken in two different view points

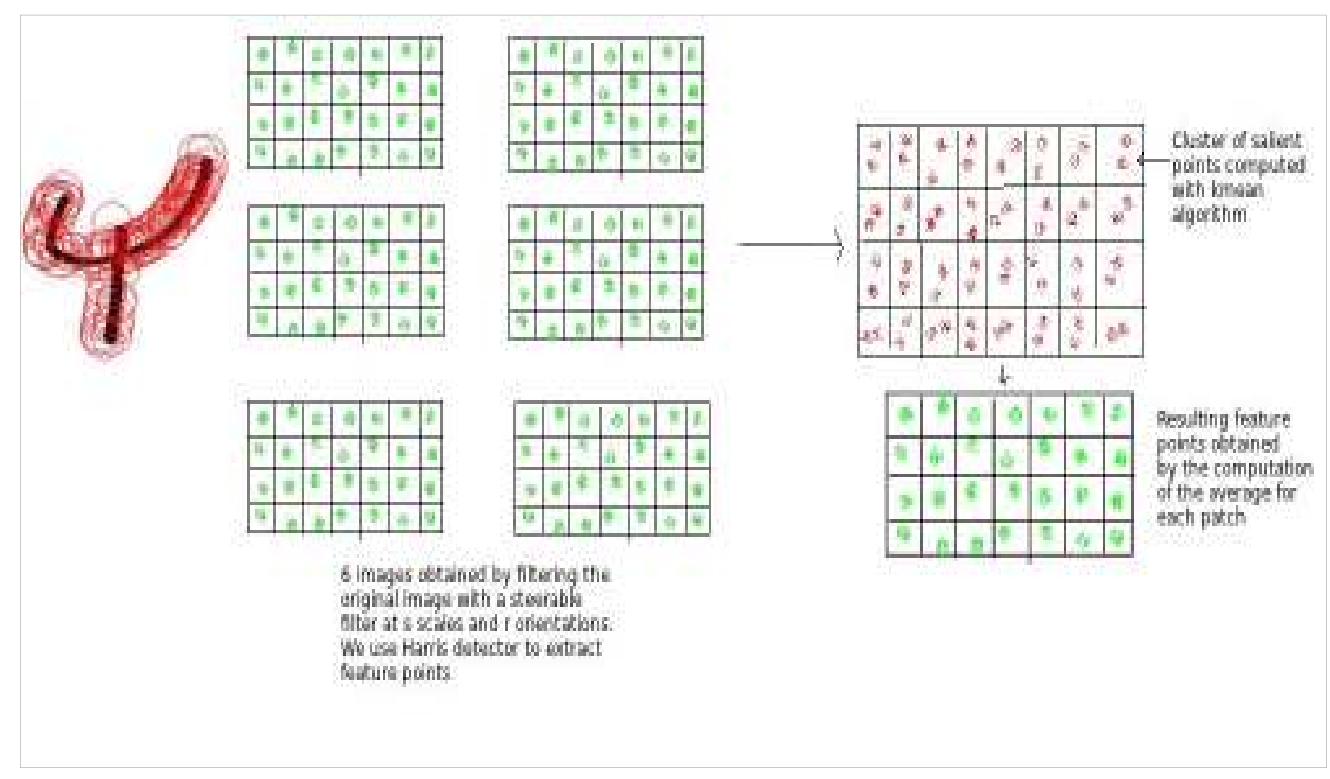

Fig5. The scheme of the RID detector descriptor 


\section{Learning}

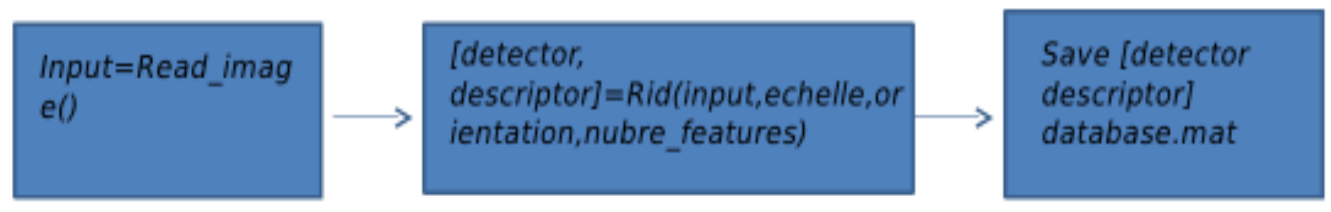

\section{Matching}

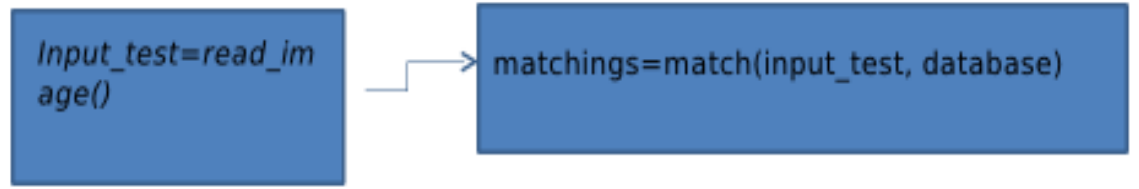

Fig6. The different modules which we use for the learning of a Tifinagh character. The matching is done with the module match. 